Z. Klin. Chem. Klin. Biochem.

11. Jg. $1973,123-127$

\title{
Zur Methodik der Ammoniakbestimmung im Urin von Früh- und Neugeborenen: \\ Vergleich zwischen Formoltitration und enzymatischer $\mathrm{NH}_{4}{ }^{+}$-Bestimmung
}

\author{
Von H.-B. v. Stockhausen und MaIke Struve \\ Kinderklinik und Poliklinik (Direktor Prof Dr. H. G. Hansen) der Medizinischen Akademie Liibeck
}

(Eingegangen am 1. August/20. Oktober 1972)

\begin{abstract}
Zahlreiche Verfahren zur $\mathrm{NH}_{4}{ }^{+}$-Bestimmung im Blut und im Urin sind bisher angegeben und von anderer Seite auch wieder kritisiert worden. Die seit einigen Jahren für die Bestimmung von Ammoniak im Blut bekannte enzymatische Methode von KIRSTEN et al. ([1963], Biochem. Z. 337, 312-319), Schmidt \& Schwarz ([1966], Klin. Wochenschr. 44, 591-592) sowie Müring et al. ([1970], Deut. Med. Wochenschr. 95, 1390-1396) wurde auf ihre Anwendbarkeit bei Urinuntersuchungen geprüft und mit der sehr einfachen Formoltitration hinsichtlich Spezifität und Reproduzierbarkeit verglichen. Die Formoltitration muß trotz guter Präzision wegen der erheblichen Beeinflussung durch Aminosäuren abgelehnt werden. Dagegen bietet sich gerade für Urinuntersuchungen von Früh- und Neugeborenen mit niedrigen $\mathrm{NH}_{4}{ }^{+}$, jedoch relativ hohen Aminosäuren-Konzentrationen die enzymatische Ammoniakbestimmung an, die bei gleicher Präzision spezifisch ist und eine sehr gute Empfindlichkeit aufweist.
\end{abstract}

\section{The determination of ammonia in the urine of premature and full-term newborns: Comparison of the formol titration and the enzymic $\mathrm{NH}_{4}{ }^{+}$determination}

Numerous methods for the determination of $\mathrm{NH}_{4}+$ in blood and urine have been described and subsequently criticized by other workers. The specificity and reproducibility of the enzymatic procedure for the determination of ammonia in blood according to KIRSTEN et al. ([1963], Biochem. Z. 337, 312-319), Scrmidx \& Schwarz ([1966], Klin. Wochenschr. 44, 591-592) and Müring et al. ([1970], Deut. Med. Wochenschr. 95, 1390-1396) was tested on urines by comparing it with the very simple formol titration method. The latter method is unsuitable in spite of its high precision, because of the marked influence exerted by amino acids. On the other hand the enzymatic $\mathrm{NH}_{4}+$ determination is applicable to the urines of premature and full-term newborns particularly because of the relatively low $\mathrm{NH}_{4}+$ level and the relatively high amino acid concentration. Its precision is equal to the formol titration method, but in addition it is specific and posesses a high sensitivity.

Zur Bestimmung von Ammoniak in biologischen Flüssigkeiten ist bis heute das Reagenz nach BERTHELoT am gebräuchlichsten $(1,2)$. Trotz wiederholter Abwandlungen, insbesondere bei Untersuchungen des Blutes, ist die Methode jedoch unbefriedigend geblieben, da die Reaktion nicht spezifisch ist und vor allem durch Aminosäuren, aber auch durch Harnsäure, Kreatinin und Sulfonamide beeinflußt wird (1-3). Auch bei Untersuchungen im Urin muß mit größeren Ungenauigkeiten gerechnet werden, wenn der Ammoniakgehalt, wie z. B. in der Neugeborenenperiode, relativ niedrig liegt, andererseits die Aminosäurenkonzentration im Urin von Frühgeborenen ein Vielfaches betragen kann (4) Zur exakten Bestimmung von Ammoniak im Blut und im Urin Neugeborener muß daher vor Durchführung der BERTHELOT-Reaktion eine Isolierung des Ammoniaks z. B. mit Hilfe der Mikrodiffusionsverfahren nach CoNWAY oder SELIGSON vorgenommen werden $(1,3,5)$. Durch alkalische Hydrolyse einiger Aminosäuren wie Glutamin, Lysin, Arginin, Alanin und Serin können aber auch bei der im übrigen relativ aufwendigen Mikrodiffusion etwas zu hohe Werte gewonnen werden $(1-3$, 5). Wobl wegen ihrer bestechenden Einfachheit ist gerade von Pädiatern die lange bekannte Formoltitration
(6) zur Bestimmung von Ammoniak im Urin wieder aufgegriffen worden, zumal man in einem Arbeitsgang die Titrationsazidität, Ammoniak und die Netto-SäureAusscheidung bestimmen kann (7-11). Andererseits ist vor einigen Jahren eine enzymatische Methode zur Bestimmung von Ammoniak im Blut von KIRSTEN et al., SCHMidt \& Schwarz sowie Müring et al. angegeben worden (12-14). Über die Verwendung dieser Methode zur $\mathrm{NH}_{4}{ }^{+}$-Bestimmung im Urin liegen unseres Wissens bisher keine Erfahrungen vor. Als Voraussetzung für eigene $\mathrm{NH}_{4}{ }^{+}$-Bestimmungen im Urin von Früh- und Neugeborenen galt es für uns zu prüfen, wie weit die enzymatische Methode für Untersuchungen im Urin anwendbar ist und im Vergleich zur einfachen, relativ verbreiteten Formoltitration zu befriedigenden reproduzierbaren Ergebnissen führt. Gleichzeitig sollte die Beeinflußbarkeit beider Methoden durch verschiedene physiologisch im Urin auftretende N-haltige Substanzen unter standardisierten Bedingungen untersucht werden.

\section{Methodik}

Enzymatische $\mathrm{NH}_{4}{ }^{+}-$Bestimmung

Prinzip

Für die enzymatische $\mathrm{NH}_{4}{ }^{+}-$Bestimmung gilt folgende Reaktionsgleichung (12-14): 
Glutamat-

dehydrogenase

2-Oxoglutarat $+\mathrm{NH}_{4}++\mathrm{H}^{+}+\mathrm{NADH} \rightleftharpoons$ Glutamat $+\mathrm{NAD}+\mathrm{H}_{2} \mathrm{O}$.

Die relativ geringe Affinität des Ammoniumions zur Glutamatdehydrogenase wird durch einen Uberschuß an Enzym ausgeglichen $(12,13)$. Bei genügend getinget Ammoniakkonzentration (unter $0,5 \mathrm{mmol} / \mathrm{l}$ ) ist diese dem gebildeten $\mathrm{NAD}$ bzw. dem gemessenen Extinktionsabfall proportional.

\section{Lösungen}

1. Triäthanolamin-Puffer $(50 \mathrm{mmol} / \mathrm{l})$ mit EDTA $(5 \mathrm{mmol} / \mathrm{l})$, $\mathrm{pH}$ 7,5:

$0,928 \mathrm{~g}$ Triäthanolamin-hydrochlorid und $0,186 \mathrm{~g} \mathrm{Na}$ EDTA werden in etwa $80 \mathrm{ml}$ Wasser gelöst und mit $\mathrm{NaOH}(4 \mathrm{~mol} / \mathrm{l})$ auf $\mathrm{pH} 7,5$ eingestellt. Anschließend mit Wasser auf $100 \mathrm{ml}$ auffüllen.

2. 2-Oxoglutarat-Lösung ( $34 \mathrm{mmol} / \mathrm{l})$ :

$50 \mathrm{mg}$ 2-Oxoglutarsäure mit etwa $9 \mathrm{ml} \mathrm{H}_{2} \mathrm{O}$ lösen, $\mathrm{pH}$ mit $\mathrm{NaOH}$

( $1 \mathrm{~mol} / \mathrm{l}$ ) auf $\mathrm{pH} 7,0$ einstellen und auf $10 \mathrm{ml} \mathrm{mit} \mathrm{H}_{2} \mathrm{O}$ auffüllen.

3. NADH-Lösung ( $3 \mathrm{mmol} / \mathrm{l})$ :

$20 \mathrm{mg} \mathrm{NADH}$ auf $10 \mathrm{ml}$ mit Triäthanolamin-EDTA-Puffer (50 mmol/l) lösen.

4. Glutamatdehydrogenase (EC 1.4.1.2) in Glycerin ( $10 \mathrm{mg} / \mathrm{ml}$ ). Sämtliche Reagenzien zum Ansatz der Lösungen wurden von der Fa. Boehringer, Mannheim, bezogen.

\section{Vorgeben}

Die Untersuchungen erfolgten zunächst mit einem registrierenden Eppendorf-Photometer, später mit einem Elko III der Firma Carl Zeiss, bei $366 \mathrm{~nm}$ und einer Küvettenschichtdicke von $1 \mathrm{~cm}$. Eine Enteiweißung des Urins war nicht erforderlich. Wegen der relativ hohen Ammoniak-Konzentration im Harn waren Verdünnungen mit Wasser bei Untersuchungen von Urinen Frühund Neugeborener im allgemeinen im Verhältnis $1: 10$ bis 1:50 und von Urinen Erwachsener $1: 100$ bis $1: 250$ notwendig. Für alle Verdünnungen sowie zum Ansatz der Lösungen wurde frisch abgekochtes demineralisiertes bidest. Wasser verwendet.

\begin{tabular}{lcc}
\hline In Küvetten wurden pipettiert & $\begin{array}{c}\text { Leerwert } \\
(\mathrm{ml})\end{array}$ & $\begin{array}{c}\text { Probe } \\
(\mathrm{ml})\end{array}$ \\
\hline $\begin{array}{l}\text { Lösung 1 (Puffer) } \\
\text { Urinprobe } \\
\text { Wasser }\end{array}$ & 2 & 2 \\
& 1 & 1 \\
\hline
\end{tabular}

gut mischen und Küvetten

verschlossen halten

\begin{tabular}{lll}
\hline Lösung 2 (2-Oxoglutarat) & 0,2 & 0,2 \\
Lösung 3 (NADH) & 0,2 & 0,2 \\
\hline
\end{tabular}

nach Durchmischung Extinktion der

Probe $\left(E_{1}\right.$ Probe) und des Leerwertes

( $E_{1}$ Leerwert) messen

\begin{tabular}{lll}
\hline Lösung 4 (Glutamatdehydrogenase) & 0,04 & 0,04 \\
\hline
\end{tabular}

Die Reaktion kommt bereits nach 15 min zum Stillstand, so daß nach 20 min der Extinktionsabfall von Probe und Leerwert abgelesen werden kann. Die Berechnung der gesuchten Substratkonzentration $\mathrm{NH}_{4}{ }^{+}$erfolgt nach den Angaben von BERGMEYER (15).

Formoltitration

Prinzip

Die Formoltitration von Ammoniak erfolgt nach der Summenformel:

$4 \mathrm{NH}_{4}+\mathrm{X}^{-}+6 \mathrm{HCHO}+4 \mathrm{Na}^{-\mathrm{OH}^{-}} \rightarrow\left(\mathrm{CH}_{2}\right)_{6} \mathrm{~N}_{4}+4 \mathrm{Na}^{+} \mathrm{X}^{-}$ $+10 \mathrm{H}_{2} \mathrm{O}$.

$\mathrm{NH}_{3}$ kondensiert mit Formalin zu der schwachen Base Hexamethylentetramin. Bei $\mathrm{pH} 7,4$ können praktisch alle Protonen des $\mathrm{NH}_{4}+$ mit $\mathrm{NaOH}$ titriert werden $(6,7)$.

\section{Vorgeben}

Die Methode wurde nach den Angaben von JörgEissen und KILDEBERG (9) unter Verwendung eines Metrohm-Kombititrators $3 \mathrm{D}$ gering modifiziert und stets bei $37^{\circ} \mathrm{C}$ durchgeführt $(7,9)$ :

a) $1 \mathrm{ml}$ der $\mathrm{zu}$ untersuchenden Probe und $0,1 \mathrm{ml} \mathrm{HCl}(1 \mathrm{~mol} / \mathrm{l})$ wurden. 5 min lang gerührt, womit der Urin bei $\mathrm{pH}<4$ frei -von $\mathrm{CO}_{2}$ und Carbonat ist; anschließend Titration mit $\mathrm{NaOH}$ $(0,2 \mathrm{~mol} / \mathrm{l})$ bis $\mathrm{pH} 7,4$

b) darauf Zugabe von $5 \mathrm{ml}$ einer Formaldehydlösung $(75 \mathrm{~g} / \mathrm{l})$, die mit $\mathrm{NaOH}$ vorher auf $\mathrm{pH} 7,4$ eingestellt wurde, sowie erneute Titration mit $\mathrm{NaOH}$ bis $\mathrm{pH} 7,4$.

c) zur Kontrolle einmalig $5 \mathrm{ml}$ Formaldehyd und $0,1 \mathrm{ml} \mathrm{HCl}$ $(1 \mathrm{~mol} / \mathrm{l}) \mathrm{mit} \mathrm{NaOH}(0,2 \mathrm{~mol} / \mathrm{l})$ bis 7,4 titrieren, um den Leerwert zu erhalten.

\section{Berechnung}

Die registrierten Titrationsmengen $a, b$ und $c$ werden zur Berechnung der Titrationsazidität, des Ammoniaks und der NettoSäure-Ausscheidung in folgende Formeln eingesetzt:

Titrationsazidität $[\mathrm{mmol} / \mathrm{l}]=\frac{2 \mathrm{a}-1}{10} \cdot 1000$

$$
\mathrm{NH}_{4}+[\mathrm{mn} ı \mathrm{l} / \mathrm{l}]=\frac{2 \mathrm{~b}-(2 \mathrm{c}-1)}{10} \cdot 1000
$$

Netto-Säure-Ausscheidung [mmol/l] $=\mathrm{NH}_{4}{ }^{+}+$Titrationsazidität.

\section{Ergebnisse}

Die beiden Methoden wurden durch Reihenmessungen an verschiedenen Urinproben und einem AmmoniakStandard $(14,5 \mathrm{mmol} / \mathrm{l})$ verglichen und ihre Präzision bestimmt. In Tabelle 1 sind die Mittelwerte $\overline{\mathbf{x}}$, ihre Standardabweichung $s$ und der Variationskoeffizient VK von je $10 \mathrm{NH}_{4}+$-Bestimmungen desselben Standards auf enzymatischem Wege und durch Formoltitration sowie von 4 verschiedenen Urinen unterschiedlichen $\mathrm{NH}_{4}^{+-}$ Gehaltes zusammengestellt. Die Reproduzierbarkeit kann danach für beide Verfahren als gleich gut angesehen werden. Der 95\%-Bereich ( \pm 2 s) liegt für beide Methoden $z$ wischen \pm 1 und $3 \%$.

Tabelle 2 zeigt die Einflüsse einer Reihe von Aminosäuren sowie von Cholin, Kreatinin, Harnstoff, Harnsäure und einer Albuminlösung auf die Bestimmung von Ammoniak in wäßriger $\mathrm{NH}_{4}^{+}$-Lösung und in einem bekannten $\mathrm{NH}_{4}{ }^{+}-$Standard. Mit Hilfe der enzymatischen Methode konnte keinmal in den $\mathrm{NH}_{4}+$-freien

Tab. 1

Untersuchung der Reproduzierbarkeit der Formoltitration sowie der enzymatischen $\mathrm{NH}_{4}{ }^{+}$-Bestimmung durch je 10 Messungen eines $\mathrm{NH}_{4}{ }_{+}$ Standards sowie 4 verschiedener Urine

\begin{tabular}{llll}
\hline \multicolumn{1}{c}{ Probe } & $\overline{\mathrm{x}}$ & $\mathrm{NH}+[\mathrm{mmol} / \mathrm{l}]$ & $\begin{array}{c}\text { Variations- } \\
\text { koeffizient } \\
\text { VK in } \%\end{array}$ \\
\hline $\begin{array}{l}\text { Ammoniakstandard } \\
\text { enzymatisch }\end{array}$ & 14,57 & 0,08 & \\
Formoltitration & 14,68 & 0,18 & 0,54 \\
\hline 1. Urin enzym. & 45,35 & 0,58 & 1,2 \\
2. Urin enzym. & 21,99 & 0,3 & 1,2 \\
3. Urin Titr. & 60,75 & 0,23 & 1,3 \\
4. Urin Titr. & 8,31 & 0,07 & 0,38 \\
\hline
\end{tabular}


Lösungen Ammoniak nachgewiesen werden. Die Formoltitration führte dagegen bei Untersuchung derselben Lösungen $\mathrm{zu}$ einem irrtümlichen $\mathrm{NH}_{4}{ }^{+}$-Gehalt, der genau dem Wert entsprach, um den die Bestimmung des $\mathrm{NH}_{4}{ }^{+}$-Standards im Vergleich zur enzymatischen Methode zu hoch ausfiel. Während Cholin; Kreatinin, Harnstoff und Harnsäure auf beide Methoden keinen Einfluß haben, kann die Anwesenheit von Aminosäuren zu erheblichen Abweichungen der Meßergebnisse bei der Formoltitration führen. Eine Umrechnung der gemessenen Differenzen auf $10 \mathrm{mmol} / 1$ Einwaage der jeweiligen Aminosäure (letzte Spalte der Tabelle 2) läßt erkennen, $\mathrm{da} B$ der Fehler in Gegenwart von Glycin und Cystein nahezu der Gesamtkonzentration der betreffenden
Aminosäure entspricht, während Prolin zu keiner Verfälschung der Ergebnisse führt. Zur Verdeutlichung des Unterschiedes zwischen den Aminosäuren Prolin und Glycin haben wir Titrationskurven der beiden Aminosäuren allein und nach Zugabe von Formaldehyd ebenfalls mit dem Metrohm-Kombititrator $3 \mathrm{D}$ aufgezeichnet (Abb. 1). Es muß betont werden, daß sich pKWerte auf diese Weise nicht exakt bestimmen lassen, dennoch entsprechen die $\mathrm{pK}_{2}$-Werte und die isoelektrischen Punkte (IP) nahezu den Angaben der Literatur $(16,17)$. Durch die große Menge an zugegebenem Formaldehyd verlaufen diese Kurven im oberen und unteren pH-Bereich flacher, zumal die gleiche Natronlaugekonzentration verwendet wurde. Dennoch unterscheiden
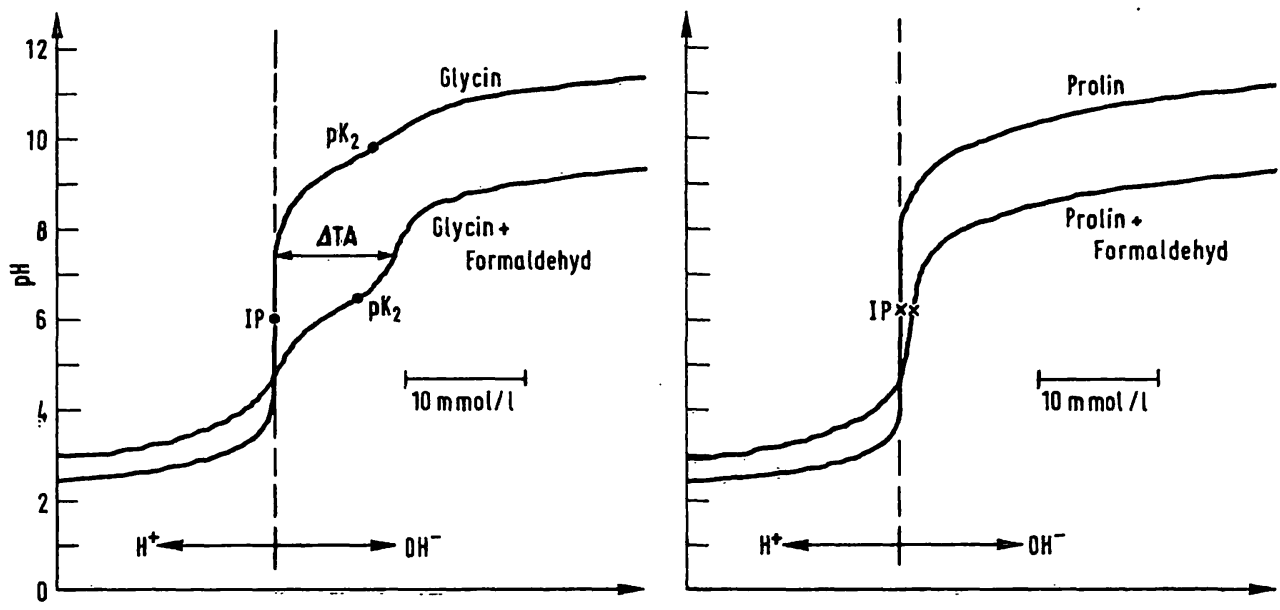

Abb. 1

Titrationskurven von Glycin und Prolin mit und ohne Zugabe von Formaldehyd

Tab. 2

Prüfung der Spezifität der enzymatischen $\mathrm{NH}_{4}{ }^{+}$-Bestimmung sowie der Formoltitration. Verschiedene Aminosäuren und einige weitere $\mathrm{N}$-haltige Substanzen wurden jeweils in wäBriger Lösung sowie in einem bekannten $\mathrm{NH}_{4}^{+}$-Standard untersucht. Die letzte Spalte zeigt eine Umrechnung der bei der Formoltitration zu beobachtenden Differenz bezogen auf $10 \mathrm{mmol} / \mathrm{l}$ eingesetzte Substanz

\begin{tabular}{|c|c|c|c|c|c|}
\hline \multicolumn{3}{|c|}{$\begin{array}{c}\text { Enzymatische } \mathrm{NH}_{4}^{+} \text {-Bestimmung } \\
{\left[\mathrm{mmol}^{-1]}\right]}\end{array}$} & \multicolumn{3}{|c|}{$\begin{array}{c}\text { Formoltitration } \\
{[\mathrm{mmol} / \mathrm{l}]}\end{array}$} \\
\hline Geprüfte Substanz & $1 \mathrm{mg} / \mathrm{ml} \mathrm{Substanz}$ & $\begin{array}{c}\mathrm{NH}_{4}^{+-} \\
\text {Standard }+1 \mathrm{mg} / \mathrm{ml} \\
\text { Substanz }\end{array}$ & $1 \mathrm{mg} / \mathrm{ml}$ Substanz & $\begin{array}{c}\mathrm{NH}_{4}^{+-} \\
\text {Standard }+1 \mathrm{mg} / \mathrm{ml} \\
\text { Substanz }\end{array}$ & $\begin{array}{l}\text { Umrechnung der } \\
\text { Differenz auf } \\
10 \mathrm{mmol} / 1 \\
\text { eingesetzte Substanz }\end{array}$ \\
\hline 1. DL-Alanin & $\varnothing$ & 14,7 & 4,2 & 18,7 & 3,74 \\
\hline 2. L-Arginin & $\varnothing$ & 14,9 & 4,5 & 18,8 & 7,8 \\
\hline 3. L-Asparagin & $\varnothing$ & 14,9 & 4,9 & 19,3 & 7,35 \\
\hline 4. L-Cystein & $\varnothing$ & 14,65 & 7,8 & 22,3 & 9,45 \\
\hline 5. Glycin & $\varnothing$ & 14,8 & 12,4 & 25,8 & 9,32 \\
\hline 6. L-Histidin-Monochlorid & $\varnothing$ & 14,7 & 0,5 & 14,9 & 1,04 \\
\hline 7. L-Leucin & $\varnothing$ & 14,7 & 2,2 & 16,8 & 2,88 \\
\hline 8. L-Methionin & $\varnothing$ & 14,7 & 4,2 & 18,7 & 6,26 \\
\hline 9. L-Phenylalanin & $\varnothing$ & 14,5 & 3,2 & 17,4 & 5,28 \\
\hline 10. L-Prolin & $\varnothing$ & 14,9 & $\varnothing$ & 14,8 & $\varnothing$ \\
\hline 12. DL-Valin & $\varnothing$ & 14,5 & 2,0 & 16,3 & 2,34 \\
\hline 13. Cholinchlorid & $\varnothing$ & 14,5 & $\varnothing$ & 14,5 & $\varnothing$ \\
\hline 14. Kreatinin & $\varnothing$ & 15,0 & $\varnothing$ & 14,6 & $\varnothing$ \\
\hline 15. Harnstoff & $\varnothing$ & 15,1 & $\varnothing$ & 14,3 & $\varnothing$ \\
\hline 16. Harnsäure & $\varnothing *$ & $15,0 *$ & $\varnothing *$ & $14,4^{*}$ & $\varnothing^{*}$ \\
\hline 17. Albuminlösung (2 g/l) & $\cdot-$ & - & $\varnothing$ & 14,4 & - \\
\hline
\end{tabular}

\footnotetext{
Wegen der schlechten Löslichkeit von Harnsäure beträgt die zugesetzte Substanzmenge nur $0,1 \mathrm{mg} / \mathrm{ml}$
} 
Tab. 3

Parallelbestimmung von Ammoniak im Frühgeborenen- und Erwachsenenurin mit Hilfe der Formoltitration und auf enzymatischem Wege zur Veranschaulichung der zu erwartenden prozentualen und absoluten Differenz des Meßergebnisses

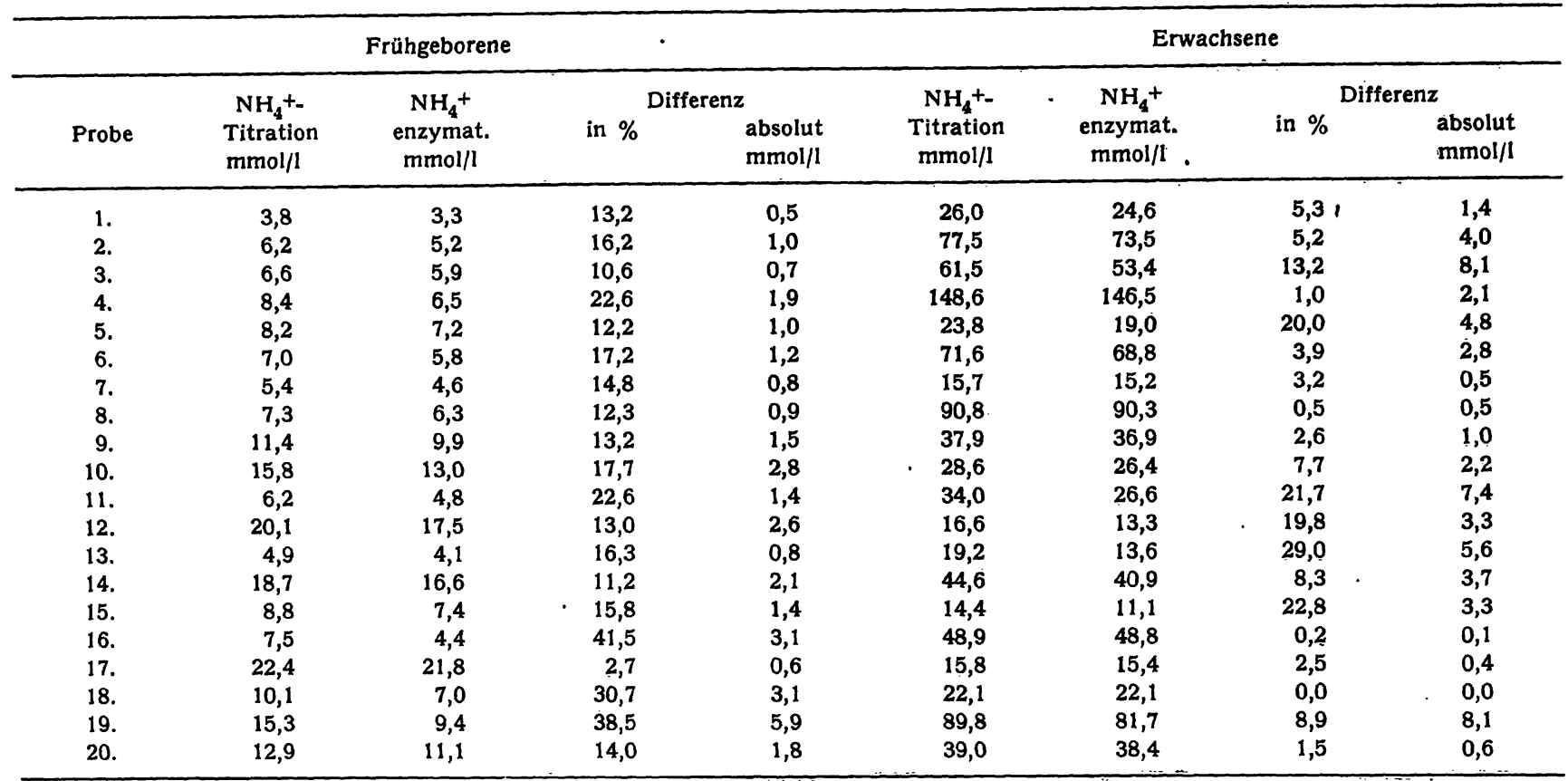

sich die Kurven für Prolin nicht wesentlich, während es beim Glycin zu einer erheblichen Verformung in Gegenwart von Formaldehyd kommt. Anhand der Titrationskurven des Glycins kann auch gezeigt werden, daß der Mehrverbrauch an Lauge $(\triangle \mathrm{TA})$ bis $\mathrm{zu}$ einem Endpunkt von $\mathrm{pH} 7,4$ in Gegenwart von Formaldehyd nahezu $10 \mathrm{mmol} / \mathrm{l}$ beträgt und damit in etwa der eingesetzten Gesamtkonzentration an Glycin entspricht (18).

In Anbetracht des starken Einflusses einzelner Aminosäuren auf die Formoltitration und der bekannten vermehrten Ausscheidung von Aminosäuren im Harn von Früh- und Neugeborenen (4) haben wir schließlich die absolute und prozentuale Differenz zwischen beiden Methoden bei je 20 Doppelmessungen von Urinen Frühgeborener und Erwachsener bestimmt (Tab. 3). Die mittlere prozentuale Differenz betrug für den Erwachsenenurin 8,9\% und für den Frühgeborenenurin $18,1 \%$. Der Unterschied ist nach dem $t$-Test mit einem $\mathrm{p}^{\circ}<0,01$ signifikant. Eine Korrelation zwischen dem absoluten Fehler und der Höhe des Ammoniak-Gehaltes im Urin bestand bei einem $r=0,168$ nicht.

\section{Diskussion}

Uns kam es darauf an, die Anwendbarkeit der enzymatischen $\mathrm{NH}_{4}{ }^{+}$-Bestimmung bei Urinuntersuchungen $\mathrm{zu}$ prüfen, da uns ihre bisher relativ geringe Verbreitung ungerechtfertigt erschien. MürING erwähnt für Untersuchungen des Blutammoniakspiegels (14), daß die Reaktion gelegentlich schleicht. Wir konnten dies bisher nicht beobachten. Die graphische Registrierung der Reaktion zeigte, daß diese bereits nach $15 \mathrm{~min}$ abge- schlossen ist. Regelmäßig wurde eine $10 \mathrm{~min}$ lange Konstanz der Extinktion beobachtet. Die Reaktion ist spezifisch und sehr empfindlich. $\mathrm{Da}$ man mehrere Proben nebeneinander ansetzen kann, ist der Zeitaufwand vor allem bei Reihenuntersuchungen kaum größer als mit der Titrationsmethode. Als kleiner Nachteil bei Urinuntersuchungen muß angesehen werden, daß die $\mathrm{NH}_{4}{ }^{+}$ Konzentration nicht über $0,5 \mathrm{mmol} / 1$ in Anbetracht der relativ geringen Affinität von Glutamatdehydrogenase zu $\mathrm{NH}_{4}{ }^{+}(12,13)$ betragen darf, so daß praktisch immer eine Verdünnung des Urins durchgeführt werden muß. Sehr exaktes Arbeiten und garantiert ammoniakfreies Wasser sind daher Voraussetzung. Kleinste Verunreinigungen werden allerdings durch die Korrektur mit einem Leerwert aufgefangen. Der Extinktionsabfall des Leerwertes sollte in der Regel nicht über 0,025 liegen und kann damit indirekt als Maßstab für fehlerfreies Arbeiten gelten. Nach KIRSTEN kann ein Extinktionsabfall des Leerwertes bis 0,013 auf Ammoniakbeimengungen der Enzym- und NADH-Lösungen zurückzuführen sein (12). Auch wenn die Formoltitration zur $\mathrm{NH}_{4}{ }^{+}$-Bestimmung im Urin bestechend einfach ist und zu sehr guten reproduzierbaren Ergebnissen führt, muß sie für Untersuchungen von Utinen mit niedriger $\mathrm{NH}_{4}{ }^{+}$ jedoch relativ hoher Aminosäurenkonzentration insbesondere bei Früh- und Neugeborenen abgelehnt werden. Hier bietet sich unseres Erachtens das enzymatische Verfahren als Methode der Wahl an, soweit nicht eine direkte $\mathrm{NH}_{4}{ }^{+}$-Bestimmung durch eine in Erprobung befindendliche $\mathrm{NH}_{4}+-$ Elektrode $^{1}$ ) die Diskussion in Zukunft in eine ganz neue Richtung lenkt.

1) Colora Meßtechnik GmbH, Lorch/Württ. 


\section{Literatur}

1. Richterich, R. (1968), Klinische Chemie, 2. Aufl., S. 253-260. Akademische Verlagsgesellschaft, Frankfurt a. M. - 2. LORENTZ, K. (1967), diese Z. 75, 291-298. - 3. LORENTZ, K. \& OssENBERG, F. W. (1967), Med. Lab. 20, 77-88. - 4. BRODEHI, J. (1969), Der renale Transport der Aminosäuren im Säuglings- und Kindesalter. Arch. Kinderheilk., Beiheft 16. - 5. RichtrRich, R. \& Cогомво, J. P. (1962), Arztl. Lab. 8, 129-141. - 6. BJöRNAndersen, H. \& Lauritzen, M. (1910), Hoppe-Seyler's $Z$. Physiol. Chem. 64, 21-38. - 7. Jörgenssen, K. (1957), Scand. J. Clin. Lab. Invest. 9, 287-291. - 8. Ballabriga, A. \& MArtinez, M. (1969), Helv. Paediat. Acta 24, 111-117. - 9. Kildeberg, P. (1968), Clinical acid-base physiology. Studies in neonates, infants, and young children, 1. Aufl., S. 31-36 Verlag Munksgaard, Copenhagen. - 10. Kildeberg, P., Enget, K. \& Winters, R. W. (1969), Acta Paediat. Scand. 58, 321-329. - 11. Oetriker,
O., Chattas, A. J. \& Schultz, S. M. (1971), Helv. Paediat. Acta 26, 523-534. - 12. Kirsten, E., Gerez, C. \& Kirsten, R. (1963), Biochem. Z. 337, 312-319. - 13. SснмIDr, F. H. \& SchWARZ, M. (1966), Klin. Wochenschr. 44, 591-592. - 14. Müting, D., Heinze, J., Momper, M. \& Schwarz, M. (1970), Deut. Med. Wochenschr. 95, 1390-1396. - 15. BergmeYer, H. U., Bernt, E., Grassl, M. \& Mrchal, G. (1970), in: Methoden der enzymatischen Analyse (BrRgMeYER, H. U. Hrsg.) Bd. 1, 273-280, Verlag Chemie, Weinheim/Bergstr. - 16. Grassmann, W., Schineider, F. \& Trupke, J. (1951), in: Lehrund Handbuch der Physiologischen Chemie (Flaschenträger, B., Hrsg.) Bd. 1, 489-584, Verlag Springer, Berlin-GöttingenHeidelberg. - 17. Raporort, S. M. (1965), Medizinische Biochemie, 3. Aufl., S. 44-47, VEB-Verlag Volk u. Gesundheit, Berlin. - 18. LevY, M. (1943), Advanc. Enzymol. 3, 454-458.

Dr. med. Hans-Burckhard v. Stockhausen Kinderklinik der Medizinischen Akademie Lübeck 24 Lübeck

Kronsforder Allee 71/73 